\title{
APTITUD DEL SUELO PARA CULTIVO DE MANGO VALLENATO EN GUACOCHE, DEPARTAMENTO DEL CESAR
}

\section{FITNESS OF A SOIL FOR GROWING VALLENATO MANGO IN GUACOCHE, CESAR STATE}

\author{
Ricardo Durán ${ }^{1 *}$, Aída L. Villa² y María T. Flórez ${ }^{3}$
}

Recibido para publicación: Enero 15 de 2014 - Aceptado para publicación: Marzo 26 de 2014

\begin{abstract}
RESUMEN
Se evaluó la aptitud del suelo de la Finca "La Estancia" ubicada en Guacoche, departamento del Cesar, para el cultivo de mango (Mangifera indica L.) variedad Vallenato. Se muestreó una hectárea de suelo con 16 calicatas para definir el perfil modal del suelo y describir sus propiedades. Se tomaron muestras disturbadas y no disturbadas de cada horizonte para análisis fisicoquímico según Manual No. 47 del Instituto Colombiano Agropecuario y la metodología establecida por el Instituto Geográfico Agustín Codazzi (IGAC). El suelo fue clasificado como un Typic Dystrustepts, arenoso franco mixto isohipertérmico, de mediana fertilidad, según Claves de Taxonomía de Suelos de los Estados Unidos. El suelo no tiene limitaciones por sales, sodio o aluminio; localmente presentan niveles freáticos altos que pueden ocasionar pudrición de la raíz y facilitar la pérdida de fertilizantes con aplicación edáfica.
\end{abstract}

Palabras clave: suelo, mango vallenato, Typic Dystrustepts, cultivo, aptitud

\begin{abstract}
The ability of the "La Estancia" farm soil in Guacoche, Cesar state, for growing Vallenato mango (Mangifera indica L.) was evaluated. One hectare of soil was sampled with 16 pits to define the modal soil profile and describe its properties. Physicochemical analysis were carried out to disturbed and not disturbed samples from each horizon under the methodology reported in the Manual No. 47 del Instituto Colombiano Agropecuario and the methodology established by the Agustín Codazzi Geographical Institute (ACGI). According to the properties and the guide of Keys to Soil Taxonomy de los Estados Unidos, the soil was classified as a Typic Dystrustepts, sandy loam mixed isohyperthermic, of medium fertility., The soil does not have limitation for salts, sodium, or aluminum; locally the soil has high water tables that can cause root rot and facilitate the loss of fertilizer with edaphic application.
\end{abstract}

Key words: Soil, mango vallenato, Typic Dystrustepts, crop, production

${ }^{1 *}$ Grupo Optimización Agroindustrial. Universidad Popular del Cesar. Campus sede Sabanas, bloque F, Lab 201. Valledupar, Colombia. Cel: 3157247875. ricardoduran@unicesar.edu.co

${ }^{2}$ Grupo Catálisis Ambiental, Departamento de Ingeniería Química, Facultad de Ingeniería, Universidad de Antioquia, Calle 70 No. 52-21, Medellín, Colombia, aida.villa@udea.edu.co,

${ }^{3}$ Grupo de Investigación GAIA, Facultad de Ingeniería, Universidad de Antioquia, Cra. 53 No. 61-30 SIU-UdeA. Torre II, Lab. 230, Medellín, Colombia, maria.florez@udea.edu.co 


\section{INTRODUCCIÓN}

El mango es una de las frutas que más se producen en las áreas tropicales y subtropicales del mundo y por ende de las más consumidas, especialmente por el interés del consumidor de ingerir alimentos más saludables y frescos (FAO 2014). De acuerdo con cifras de la Organización de las Naciones Unidas para la Agricultura y la Alimentación (FAO 2014), la producción mundial de mango representa aproximadamente el $36 \%$ del monto mundial de frutas tropicales, con un crecimiento anual del 1,76\%. A nivel mundial, se producen aproximadamente 36,3 millones de toneladas por año de mango (4,9 millones de hectáreas), distribuidas en Asia (79\%), América (13\%), África, Europa y Oceanía (8\%) (CCl 2009).

El comercio internacional, prevé un aumento de 1.559 millones de dólares en 2014 a 1.798 millones de dólares en 2029; por lo tanto, se proyecta en Colombia que de 18.573 ha de mango sembrada en el 2011 se pase a 40.000 en el 2020 para una producción de 266.693 t. (Plan de Fomento Hortofrutícola 2006). El departamento del Cesar, identificado como región potencial para la producción del mango (Programa de Transformación Productiva 2013) tiene un total de 949 ha de mango en cultivos, con una producción de 16.575 t y un rendimiento de 17,5 t ha $^{-1}(\mathrm{CCl} 2011)$, correspondiendo más del $50 \%$ a mango Vallenato, el cual es originario de Colombia, específicamente del departamento del Cesar, en honor a los habitantes de la ciudad de Valledupar y a la música característica de esta región (Fernández y Reales 2011).

El árbol del mango Vallenato es grande $y$ produce muy buena floración en latitudes tropicales y subtropicales. El fruto tiene forma oval con un pequeño pico, con longitud de 8 a $10 \mathrm{~cm}$, ancho de 7,5 a 8,5 cm y la piel es de espesor medio. El peso varía de 175 a 350 g, con un promedio de $250 \mathrm{~g}$, el peso de la piel y la semilla varía de 16 a $24 \mathrm{~g}$. Tiene color rojo luminoso con un color amarillo tierra en el momento de cosecha. La piel es lisa y resistente a la abrasión, con textura muy firme. La pulpa es de color amarillo-naranja con un sabor dulce, picante, firme y casi libre de fibra. La fruta es resistente a la enfermedad antracnosis y tiene excelentes características para precosecha y poscosecha. Su principal defecto es que es una fruta pequeña (CENICAFÉ y SENA 2001).

El mango se puede adaptar en alto grado a diversas condiciones edáficas y de fertilidad; sin embargo, aunque es tolerante a los suelos de baja fertilidad, sus niveles de producción se elevan sustancialmente en suelos fértiles (Avilán et al. 1998; Centa 2002).

El objetivo de este trabajo es conocer la aptitud del suelo para el establecimiento del cultivo de mango vallenato en la finca "La Estancia".

\section{MATERIALES Y MÉTODOS}

El estudio se realizó en un suelo cultivado con mango en el departamento del Cesar, municipio de Valledupar, corregimiento Guacoche, finca "La Estancia" (Coordenadas planas: N 1.094.432 - W 1.652.041). Se muestreó una hectárea de suelo utilizando una malla o cuadricula de $20 \mathrm{~m}$ x 20 m, eliminando los bordes, para evitar interferencias en el muestreo para un total de 16 puntos. En una calicata modal se describieron las propiedades físicas del suelo, en forma detallada los perfiles del suelo y se definió el 
perfil modal con sus respectivas propiedades diagnósticas. De cada horizonte se tomó 1 $\mathrm{kg}$ de suelo disturbadas y no disturbadas (en cilindro de $30 \mathrm{~cm}$ y $4 \mathrm{~cm}$ de diámetro), ambas fueron guardadas en bolsas plásticas con cierre hermético, para análisis fisicoquímicos de cada uno de los horizontes. El cilindro se cortó a lo largo en dos, una mitad se guardó como testigo y la otra mitad se utilizó para describir los perfiles con mayor detalle. El suelo se clasificó con base en la Key Taxonomy del 2010 y se comparó con el indicado en el estudio general de suelos levantado en la Sierra Nevada de Santa Marta por el IGAC (1986).

En cada horizonte se describió el espesor, color, textura, profundidad efectiva, presencia de líticos, estructura, pegajosidad, plasticidad, bioturbación, presencia y tipo de raíces y organismos (Tabla 1). Con las muestras disturbadas y secadas al aire, se evaluó la textura, $\mathrm{pH}$, conductividad eléctrica, materia orgánica, fósforo $(\mathrm{P})$ disponible, bases totales (Ca, K, Mg y $\mathrm{Na}$ ) aluminio intercambiable y los elementos menores: Fe, Cu, Mn, Zn, B. Se siguió la metodología establecida por el Instituto Geográfico Agustín Codazzi (IGAC 1990) y el Manual No. 47 del Instituto Colombiano Agropecuario (ICA 1989).

Los análisis se realizaron por triplicado, realizando promedio aritmético y desviación estándar para el primer horizonte. La evaluación de la aptitud se realizó teniendo en cuenta aspectos relacionados con el suelo (textura, profundidad efectiva, porcentaje de saturación, pedregosidad, pendiente, minerales, $\mathrm{pH}$ y materia orgánica), con el crecimiento de las plantas (disponibilidad de nutrientes, calidad del sistema radicular y disponibilidad del agua) y con pérdida de la capacidad (erosión eólica, hídrica y lixiviación) (Delgado y Sánchez 1987; Oviedo et al. 2012).

\section{RESULTADOS Y DISCUSIÓN}

\section{Aspectos relacionados con el suelo}

Este un suelo con una pendiente menor del 3\%, ubicado en la margen izquierda del rio Guatapuri, tiene características de régimen de humedad ústico, régimen de temperatura isohipertérmico superior a $30{ }^{\circ} \mathrm{C}$, un epipedón ócrico, con contenidos de materia orgánica menor a 1\%, material parental proveniente de sedimentos aluviales, un endopedón cámbico, presencia de horizontes franco arenosos; mineralogía mixta; con altos contenidos de bases, el cual se clasificó como Typic Dystrustepts arenoso franco mixto isohipertérmico, asociación Guacoche - "La Estancia", (USDA 2010) diferente a lo encontrado por el estudio de suelos del Instituto Agustín Codazzi, IGAC (1986), a escala de 1:100.000, que clasifica este suelo dentro de la Asociación Guatapurí, conjunto Guatapurí, como Typic Haplaquoll, poco desarrollado, superficial, limitado por drenaje natural pobre, nivel freático alto y encharcamiento, presencia de cantos rodados y bajo contenido de fósforo y potasio, localizados en el valle del río Guatapurí, a menos de 300 msnm, con clima cálido seco con relieve plano surcado por muchos cauces y arroyos, con problemas de inundación en épocas de lluvia.

Posiblemente debido a cambios introducidos por el uso intensivo con cultivos de arroz y frutales, cambios en los parámetros de clasificación relacionados con el régimen de humedad y temperatura del suelo (régimen de humedad ústico y régimen de temperatura isohipertérmico) y el nivel de detalle o de escala utilizada. 


\section{Propiedades físicas y químicas del suelo}

En la tabla 1, se describe el perfil modal y se detallan cada uno de los horizontes. El perfil modal está constituido desde el techo o parte superior por un horizonte $A$ de $16 \mathrm{~cm}$ de espesor, con textura franco, pedregoso con cantos de rocas de hasta $5 \mathrm{~cm}$ de diámetro en un $10 \%$ del horizonte y estructura de bloques subangulares fuertes; raíces medias y con bioturbación. Subyaciendo este se encuentra el Horizonte B de $36 \mathrm{~cm}$ de espesor, de textura franco arenoso, pedregoso con cantos de roca pequeños en una proporción del $1 \%$, estructura de bloques subangulares fuertes, no plástico, ligeramente pegajoso y alta bioturbación. Subyaciendo estos dos horizontes (52 cm en adelante) se encuentra el material parental que está constituido por un depósito aluvial muy pedregoso, producto de las inundaciones del río, en el cual son comunes los cantos heterométricos y heterogéneos de roca de 15 y $20 \mathrm{~cm}$ de diámetro, matriz soportados, en una proporción del $70 \%$ del horizonte y el resto de material es arena. La distribución no es homogénea en todos los puntos muestreados, ya que en algunos bajos del lote, el nivel freático está a $30 \mathrm{~cm}$ y en los demás a 70-80 cm.

El análisis de las propiedades químicas (Tabla 2), indica que este suelo es neutro con $\mathrm{pH}$ promedio de 7,2 $\pm 0,9$ con algunos puntos de muestreo superiores a 8 y no existe presencia de aluminio intercambiable. La materia orgánica (M.O.) es baja entre 0,57 y 1,14\%; los valores de fósforo disponible varían entre 28,7 y 160,7 mg kg-1, que corresponden a valores entre medios a muy altos, y los de potasio son medios entre 0,25 $\pm 0,1 \mathrm{cmol} \mathrm{kg}^{-1}$, requiriendo la suplementación nutricional con nitrógeno $(\mathrm{N})$, fósforo $(\mathrm{P})$ y potasio (K) en algunas etapas del cultivo. No se presentan limitaciones por sodio $(\mathrm{Na})$ y sales, con contenidos menores a $1 \mathrm{cmol} \mathrm{kg}^{-1} \mathrm{y}$ $0,73 \mathrm{dS}^{-1}$ respectivamente, ya que el mango es considerado sensible a condiciones salinas, dando lugar a puntas quemadas, deformación de las hojas, y reducción de crecimiento (Durán et al. 2003, 2004). Los valores de magnesio (Mg) son bajos $\left(0,94 \pm 0,31 \mathrm{cmol} \mathrm{kg}^{-1}\right)$ y los niveles de calcio (Ca) son altos $\left(19,0 \pm 7,9 \mathrm{cmol} \mathrm{kg}^{-1}\right)$. Los elementos menores presentan valores altos en todos los puntos, con excepción del boro (B) disponible que se encuentra entre 0,21 y 0,22 $\mathrm{mgkg}^{-1}$, o sea niveles bajos, que puede causar problemas como la muerte del centro del crecimiento o el desarrollo lento de las raíces (Malavé 2005).

En la tabla 3, se presenta el consolidado de los contenidos nutricionales del suelo en el primer horizonte. Como se evidencia, existen variaciones menores al $15 \%$ en casi todas las propiedades, con excepción de fósforo, calcio y magnesio, originado por la génesis del suelo, su interacción con las condiciones de inundación que no son homogéneas y posiblemente por la fertilización realizada en años anteriores.

\section{Aspectos relacionados con el crecimiento de las plantas}

El suelo analizado presenta estructura en bloques subangulares, con texturas franco a franco arenosas, que permiten y facilita el desarrollo de las raíces y el transporte de agua y nutrientes. Con respecto a la extracción de nutrientes, analizando frutos en un elevado número de variedades, evidencian que el nitrógeno, el fósforo y el potasio son los elementos extraídos en mayores cantidades en una cosecha, seguido del calcio y el magnesio (Medina et al. 2014). Esto indica que el suelo en estudio requiere fertilización suplementaria, para incrementar los 
Tabla 1. Descripción del perfil modal del suelo finca "La Estancia"

\begin{tabular}{|c|c|}
\hline Nombre del suelo & Finca "La Estancia" - Vereda Guacoche \\
\hline Fecha de descripción & Marzo 7 de 2009 \\
\hline Descrito por & Ricardo Durán Barón \\
\hline Localización & Coordenadas planas: N 1094432 - W1652041 \\
\hline Relieve & Plano con pendiente menor del 3\% \\
\hline Material parental & Sedimentos aluviales del río Guatapurí \\
\hline Profundidad & Suelos poco profundos con limitantes producidos por el material parental de origen aluvial. \\
\hline Drenaje natural & Imperfecto \\
\hline Vegetación natural & Rastrojo \\
\hline Uso del suelo & Cultivo de mango Vallenato \\
\hline Erosión & Hídrica \\
\hline Influencia humana & Escasa \\
\hline Observaciones & Descripción para proyecto tesis doctoral \\
\hline Epipedón & Ocrico \\
\hline Endopedón & Cambico \\
\hline Régimen de humedad & Ústico \\
\hline Régimen de Temp. & Isohipertérmico \\
\hline Orden & Inceptisol \\
\hline Grupo & Ustepts \\
\hline Gran grupo & Dystrustepts \\
\hline Serie & Typic Dystrustepts - Arenoso, franco \\
\hline Horizonte y espesor & Descripción \\
\hline Ap1 $(0-2 \mathrm{~cm})$ & $\begin{array}{l}\text { Color 10YR6/4, pardo amarillento claro. Sin estructura. Textura Franca. No plástico, no pegajoso } \\
\text { Abundantes raíces finas y gruesas. Materia orgánica media Límite inferior irregular difuso. }\end{array}$ \\
\hline Ap2 $(2-14 \mathrm{~cm})$ & $\begin{array}{l}\text { Color 10YR6/3 pardo claro. Estructura en bloques subangulares grandes y fuertes. Textura franca, } \\
\text { no plástico y ligeramente pegajoso. Abundantes raíces finas, algunas medias y gruesas. Bioturbado. } \\
\text { Presencia de material lítico hasta de } 5 \mathrm{~cm} \text { en un } 10 \% \text { del horizonte muy localizado. }\end{array}$ \\
\hline Bw $(14-50 \mathrm{~cm})$ & $\begin{array}{l}\text { Color 10YR6/4 pardo. Estructura en bloques subangulares. Textura franco arenoso, no } \\
\text { plástico, ligeramente pegajoso. Presencia de raíces gruesas, medias y finas. Alta presencia de } \\
\text { microorganismos. Abundante bioturbación. }\end{array}$ \\
\hline C1 $(50-64 \mathrm{~cm})$ & $\begin{array}{l}\text { Color } 10 \text { YR6/3 pardo claro. Sin estructura, masivo. Textura franco arenoso, no plástico, ligeramente } \\
\text { pegajoso. Presencia de raíces gruesas y fragmentos líticos subangulares desde } 3 \text { hasta } 11 \mathrm{~cm} \text { de } \\
\text { diámetro, en un } 20 \% \text { del horizonte. }\end{array}$ \\
\hline C2 $(64-100 \mathrm{~cm})$ & $\begin{array}{l}\text { Color 10YR6/3 pardo claro. Sin estructura. Pocas raíces. Abundante presencia de material de } \\
\text { arrastre con cantos de roca de hasta } 15-20 \mathrm{~cm} \text { en un } 70 \% \text { del horizonte matriz soportado, matriz } \\
\text { arenosa gruesa. Aflora el nivel freático. }\end{array}$ \\
\hline
\end{tabular}




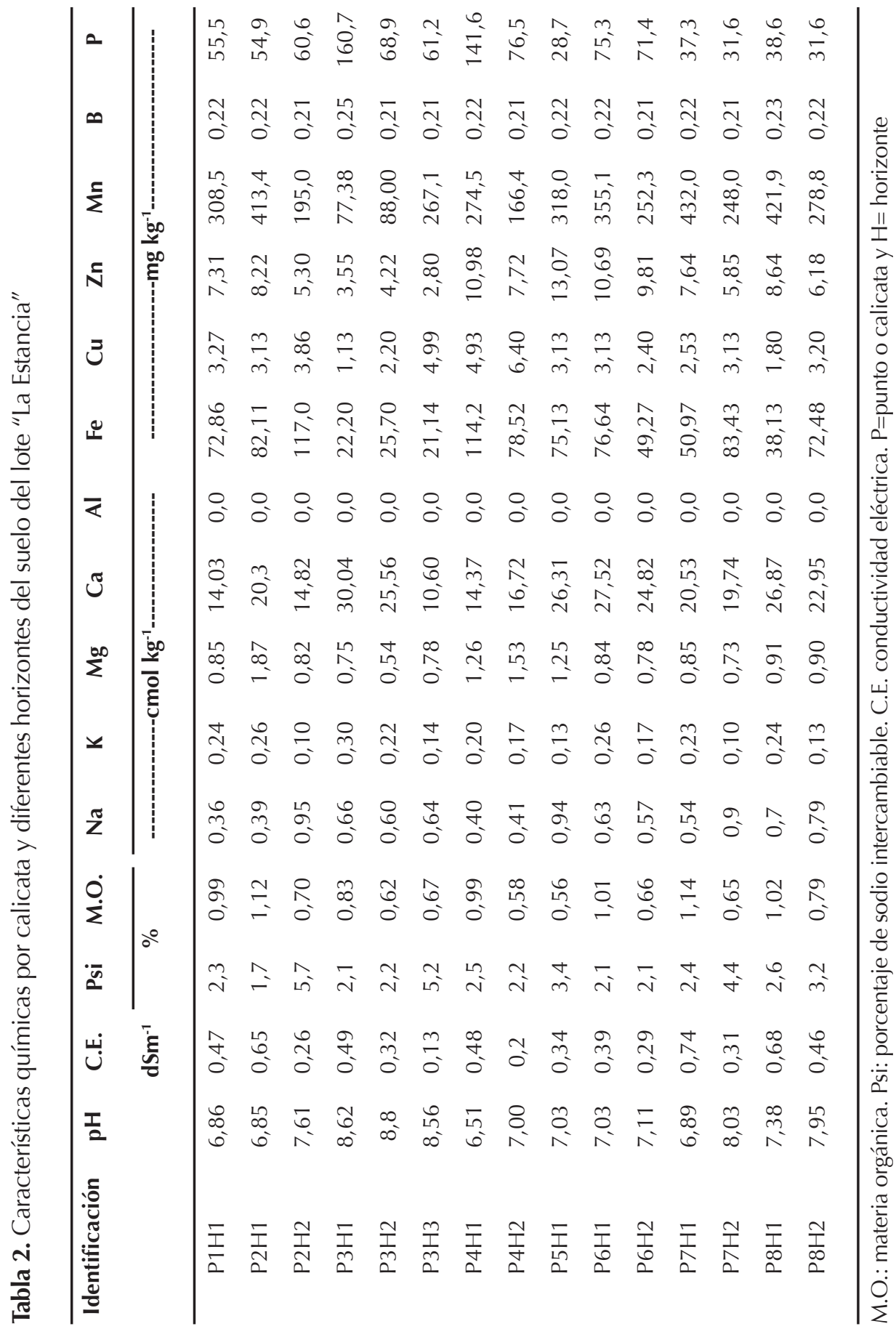

niveles de nitrógeno, fósforo, potasio, magnesio y boro para mejorar los niveles de producción, ya que el nitrógeno tiene gran influencia sobre el crecimiento y desarrollo del mango, como ingrediente esencial de clorofila, proteínas, hormonas, enzimas y la producción del fruto. El fósforo es importante en el crecimiento de raíces, tallos fuertes, frutas buenas y temporizador de la madurez y el potasio mejora la capacidad de la planta para soportar condiciones extremas tales como la sequía, el calor, la salinidad y las enfermedades y en la fruta influye en la colora- 
Tabla 3. Propiedades químicas del suelo "La Estancia" en el primer horizonte

\begin{tabular}{|c|c|c|}
\hline Propiedad & Valor promedio & Metodologías (ICA 1989) \\
\hline $\mathrm{pH}$ & $7,2 \pm 0,9$ & Potenciometría, relación $1: 1$ con agua \\
\hline Contenido de materia orgánica, \% & $0,97 \pm 0,11$ & Walkley black \\
\hline P, ppm & $74,0 \pm 36,5$ & Bray II \\
\hline Conductividad eléctrica, dS $\mathrm{m}^{-1}$ & $0,53 \pm 0,11$ & Conductimetría \\
\hline Al intercambiable, $\mathrm{cmol} \mathrm{kg}^{-1}$ & $0,0 \pm 0,0$ & Titulación \\
\hline Na intercambiable, $\mathrm{cmol} \mathrm{kg}^{-1}$ & $0,59 \pm 0,21$ & Acetato de amonio $1 \mathrm{M}$ y $\mathrm{pH} 7,0$ \\
\hline $\mathrm{K}$ intercambiable, $\mathrm{cmol} \mathrm{kg}^{-1}$ & $0,25 \pm 0,1$ & Acetato de amonio $1 \mathrm{M}$ y $\mathrm{pH} 7,0$ \\
\hline Mg intercambiable, $\mathrm{cmol} \mathrm{kg}^{-1}$ & $0,94 \pm 0,31$ & Acetato de amonio $1 \mathrm{M}$ y $\mathrm{pH} 7,0$ \\
\hline Ca intercambiable, $\mathrm{cmol} \mathrm{kg}^{-1}$ & $19,0 \pm 7,9$ & Acetato de amonio $1 \mathrm{M}$ y $\mathrm{pH} 7,0$ \\
\hline Sumatoria de bases, $\mathrm{cmol} \mathrm{kg}^{-1}$ & $20,78 \pm 7,3$ & Estimado \\
\hline Relación calcio/magnesio & 20,2 & Estimado \\
\hline Sodio intercambiable, \% & $2,4 \pm 0,2$ & Estimado \\
\hline Saturación de aluminio, \% & $0,0 \pm 0,0$ & Estimado \\
\hline $\mathrm{Fe}, \mathrm{mg} \mathrm{kg}^{-1}$ & $66,5 \pm 28,02$ & Olsen modificado \\
\hline $\mathrm{Cu}, \mathrm{mg} \mathrm{kg}^{-1}$ & $2,88 \pm 0,94$ & Olsen modificado \\
\hline $\mathrm{Zn}, \mathrm{mg} \mathrm{kg}^{-1}$ & $8,7 \pm 2,4$ & Olsen modificado \\
\hline $\mathrm{Mn}, \mathrm{mg} \mathrm{kg}{ }^{-1}$ & $325,1 \pm 97,6$ & Olsen modificado \\
\hline Boro, $\mathrm{mg} \mathrm{kg}^{-1}$ & $0,25 \pm 0,02$ & Azometina-H \\
\hline$\%$ arena & 64,0 & Hidrómetro \\
\hline$\%$ limo & 19,0 & Hidrómetro \\
\hline$\%$ arcilla & 17,0 & Hidrómetro \\
\hline
\end{tabular}

ción de la cáscara, aroma, tamaño y duración de la fruta (Ramírez y Davenport 2010).

\section{Aspectos relacionados con la pérdida de la capacidad}

El suelo presenta niveles freáticos muy altos que pueden ocasionar pudrición de la raíz, favorecer las enfermedades y facilitar la pérdida de fertilizantes por lixiviación que se apliquen a nivel edáfico. Generalmente, los árboles de mangos toleran las condiciones húmedas e inundaciones; no obstante, la respuesta es variable entre los árboles. (García et al. 2009). Los síntomas del estrés causado por las inundaciones incluyen desecación y marchitez de las hojas, muerte progresiva y crecimiento reducido. Las condiciones ambientales previas y actuales así como el tamaño y salud de los árboles afectarán la respuesta de los mismos a las condiciones húmedas (Centa 2002).

\section{Evaluación de la aptitud}

Este suelo no presenta limitaciones con respecto a las variables agroecológicas óptimas para la producción de mango, pues se encuentra a una altitud de 200 msnm; temperatura de $28,8{ }^{\circ} \mathrm{C}$; precipitación de $1000 \mathrm{~mm} / \mathrm{año}$; vientos de 1,2-2,5 $\mathrm{km} \mathrm{h}^{-1} ; \mathrm{pH}$ de 7,2 $\pm 0,9$; textura franco 
arenosa, porcentaje de sodio intercambiable de 2,1-5,2; no presenta aluminio; y los valores óptimos según (Avilán 1998) son altitud hasta 800 msnm; temperatura entre 26-32 ${ }^{\circ} \mathrm{C}$; precipitación entre 1000-1500 mm/año; vientos menores de $20 \mathrm{~km} \mathrm{~h}^{-1}$; $\mathrm{pH}$ neutro; textura arenosa hasta franco, porcentaje de sodio intercambiable hasta 15 y saturación de aluminio hasta 30.

En ciertas edades del cultivo, requeriría riego complementario en periodos de sequia (Mora et al. 2002), necesidad de drenajes para disminuir la lixiviación de nutrientes o la pudrición de raíces y suplemento nutricional en ciertas edades del cultivo.

\section{CONCLUSIONES}

El suelo es apto para el cultivo de mango vallenato, no presenta limitaciones por sales, sodio o aluminio pero si presenta limitaciones por niveles freáticos altos, en algunos sectores, que pudieran ocasionar la pudrición de la raíz y facilitar la pérdida de fertilizantes con aplicación edáfica, se hace necesario el riego complementario en periodos de sequia en ciertas edades del cultivo.

El suelo presenta las condiciones agrológicas y ambientales requeridas para el cultivo de mango Vallenato, pero podría tener limitaciones por $\mathrm{pH}$ en algunos puntos de muestreo y necesidad de fertilización suplementaria para incrementar los niveles de producción.

\section{REFERENCIAS}

Avilán, L., Rodríguez, M. y Ruiz, J. 1998. Variedades. El cultivo del manguero en Venezuela. Fondo Nacional de Investigaciones Agropecuarias. Editorial
FONAIAP. Maracay. Venezuela. p185196.

Cenicafe-Sena. 2001. Caracterización física y química de mango vallenato en el departamento del Cesar.

\section{Corporación Colombia Internacional (CCI).2009. Proyecto Mango de Exportación.}

\section{Corporación Colombia Internacional (CCI).} 2011. Encuesta Nacional Agropecuaria (ENA). 2011.

Delgado, G. y Sánchez, M. 1987. Metodología para la evaluación de la aptitud de los suelos del sector noroccidental de sierra nevada (granada) para usos agronómicos (agrícolas, forestales y ganaderos). Ecología 1:5-25.

Durán, Z., Martínez, R. and Aguilar, R. 2003. Salt tolerance of mango rootstock (Mangifera indica L. cv. 'Osteen'). Spanish Journal Agriculture Research 1: 67-78.

Durán, Z., Martínez, R. and Aguilar, R. 2004. Impact of salinity on the fruit yield of mango (Mangifera indica L. cv. 'Osteen'). European. Journal Agronomy 21:323-334.

Fernandez A. and Reales, J. 2011. Physicochemical evaluation of Mangifera indica L. fruits C.v Vallenato preserved in modified atmosphere to extend its quality. Revista Técnica de Ingeniería de la Universidad del Zulia 34(1):31-38. 
Garcia, J., Sandoval, A., Forero, F., Floriano, J., Bernal, J., Vasquez, L., Salamanca, G. y Goméz, G. 2009. Atributos de calidad del mango criollo para la agroindustria. Boletín técnico. Corpoica, C.I. Nataima, Tolima.

\section{Centro Nacional de Tecnología Agropecuaria}

y Forestal (Centa). 2002. Guía Técnica Cultivo de Mango. San Salvador, El Salvador.

Instituto Colombiano Agropecuario (ICA). 1989. Manual No. 47.

\section{Instituto Geográfico Agustín Codazzi, IGAC.} 1986. Estudio General de suelos de la Sierra Nevada de Santa Marta.

\section{Instituto Geográfico Agustín Codazzi, IGAC.} 1990. Métodos analíticos de laboratorios de suelos.

Keys to Soil Taxonomy. 2010. United States Department of Agriculture (USDA). Soil Survey Staff. 2010. 11th ed. USDA Natural Resources Conservation Service, Washington DC. 339 p.

Malavé, A. 2005. Los suelos como fuente de Boro. Revista UDO agrícola 5(1):10-26.

Medina, J., Volke v., Cortés, J., Galvis, A., González, J. y Santiago, M. 2014. Estado nutrimental y producción de fruto de mango Mangifera indica I.), cv. tommy atkins, en suelos luvisoles del estado de campeche, méxico. Revista chapingo serie horticultura 20(3): 253-268.

Mora, J., Gamboa, J. y Elizondo, R. 2002. Guía para el Cultivo de Mango. Ministerio de Agricultura y Ganadería. San José de Costa Rica.

Organización de las naciones unidas para la alimentación y la agricultura (FAO). Agronoticias América Latina y el Caribe. http://www.fao.org/agronoticias/agronoticias/detalle/en/c/159358/ [18 de Noviembre 2014].

Oviedo, M., Puerta, O., Bru, S., Atencio, V. y Pardo, S. 2012. Aptitud del Suelo de la Zona Costera del Departamento de Córdoba (Colombia) para la Piscicultura. Revista Facultad Nacional de Agronomía de Medellín 65(1): 6431-6438.

Plan de fomento hortofrutícola 2012-2022. 2006. Ministerio de Agricultura $y$ Desarrollo Rural.

Programa de Transformación Productiva. 2013. Elaboración y acompañamiento del Plan de Negocios para el Sector Hortofrutícola en Colombia. FASE IV. Plan de Negocio Mango.

Ramírez, F. and Davenport, T. 2010. Mango (Mangifera indica L.) flowering physiology. Scientia Horticulturae 126:65-72.

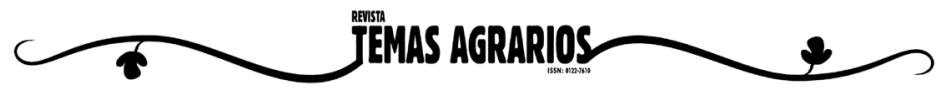

